\title{
Development of a receiver circuit for medium frequency shift keying signals.
}

\author{
Olumuyiwa Oludare FAGBOHUN \\ Department of Electrical \& Electronic Engineering, Ekiti State University, PMB 5363, Ado-Ekiti, Nigeria.
}

\begin{abstract}
Frequency shift keying (fsk) mode of digital signal information transfer switches between two predetermined frequencies of the carrier wave, either by modulating one sine wave oscillator or by switching between two oscillators. The need for a receiver to decode an fsk signal along the transmitting medium from a digital source code within about 5 kilometer radius for security monitoring of environment informed this work. The design of a receiver circuit at a frequency of $500 \mathrm{kHzfor}$ an input frequency shift keying (fsk) signal from a transmitter is presented. The receiver is to receive an RF signal, amplify it, filter it to remove unwanted signals, and recover the desired base band information. It consists of an amplifier, tuned circuitsand mixers which filters the base-band information. A comparator circuit is incorporated, to detect the digital signal received. The output from the comparators is the digital equivalent of the coded signals sent by the transmitter circuit, and transferred to a microcontroller circuit, to act as a coded signal representing information from the transmitting end. The bode-plot response of the receiver to the incoming signals using a FET tuned circuit, shows that only frequencies above $470 \mathrm{kHz}$, and below $495 \mathrm{kHz}$ are allowed to pass through the network with a resonant frequency of $483.553 \mathrm{kHz}$ and a gain of $27.734 \mathrm{~dB}$, while others are totally attenuated. The reliability of the designed receiver circuit was evaluated for a 1 year continuous operating period and was found to be 74.7\%.Area of application of this work include electronic policing of a defined environment with good success.
\end{abstract}

Keywords: Band-pass network, Comparator, Detector, FET amplifier, FSK signals, Resonant circuit, Quality factor.

\section{Introduction}

A receiver is the destination end of any communication system, having the capability of recovering the original information sent. The first three blocks of a generic receiver are the RF amplifier, mixer and oscillator[1,2]. They form what is known as the "front end" of the receiver as shown in Figure 1. The front end is very important in setting the noise figure of the receiver. In particular, the RF amplifier should be a low noise design because it amplifies all the low-level signals from the antenna - including noise - with a significant amount of gain. The better the low-noise design of the RF amplifier, and the mixer, the better the receiver sensitivity $[1,3,4]$. The RF amplifier designed to amplify the low level signal from the antenna. At the input terminal of the amplifier, a rhombic antenna was connected which receives the signals and other surrounding signals which needed to be separated. A FET amplifier was selected to amplify the received signals from the antenna end. The choice was based on its advantages over bipolar transistors which include (i) extremely high input impedance (ii) less noise and better thermal stability $[3,5]$.

The receiver circuit consists of an AM receiver and a comparator circuit. The basic purpose of a receiver is to receive an RF signal, amplify it, filter it to remove unwanted signals, and recover the desired base band information. Since the RF signal usually comes from an antenna, its amplitude is very small, often in the order of a few micro-volts $[4,6,7]$. The receiver is to amplify the signal from a very small level up to usable levels of several volts or more. The basic digital modulation techniques used in wireless communications include the frequency shift keying (fsk) among others $[6,8]$, and fsk is a form of frequency modulation in which the modulating wave shifts the output between two pre-determined frequencies, usually termed the mark and space frequencies. Because of the narrow bandwidths in fsk, it offers only slightly improved noise performance over the AM two-tone modulation scheme, however, the greater number of sidebands transmitted in fsk allow better ionospheric fading characteristics than do a two-tone AM modulator schemes. With fsk, there is a change in the output frequency each time the logic condition of the binary input signal changes. Consequently, the output rate of change is equal to the input rate of change. In digital modulation, the rate of change at the right to the modulator is called the bit rate and has the units of bits per second (bps). The rate of change at the output of the modulator is called baud rate or band and is equal to the reciprocal of the time of one output signaling element $[6,7]$. In fsk, the input and output rates of change are equal and thus, the bit rate and baud rate are equal. Recall that we modulate a sine wave carrier with the baseband binary stream of necessity to shift the resultant modulated signal to an appropriate frequency for transmission $[8,9]$. At the receiver we must undo this process or demodulate the signal to recover the original binary stream. This process of demodulation is often called detection. There are essentially two common methods of demodulation [10]. One, called 
synchronous or coherent detection, simply consists of multiplying the incoming signal by the carrier frequency that is locally generated at the receiver and then low-pass filtering the resultant multiplied signal. The other method is called envelope detection. The synchronous detection procedure in which fsk signals require two sine waves, one for each frequency transmitted is used for this work.

\section{Materials and Method}

2.1. Materials Used: An op-amp in summing mode and a FET transistor $2 \mathrm{~N} 4416 \mathrm{~A}$ as the amplifier circuit with frequency tuning were used in the design with calculations made to select the circuit parameters. A diode 1N914 was used as a detector and an fsk mode of frequency modulation was developed using two separate band pass filters with an op-amp LF411CN. A comparator TLC 372D was used to generate the digital circuit. Multism -12 was used for the design schematics and analysis of the circuit with measurements using Oscilloscopes and Bode plotter.

\subsection{Receiver circuit design for frequency shift keying signals.}

A tuned circuit amplifier offers a way to achieve gain and a particular response at high frequencies. The amplifier circuit pass-band can be controlled by the design of a resonant circuit as long as the transistor has the necessary gain. The tuned circuit for the FET amplifier was designed using equations 1 and 2 , it is a narrow band filter that passes frequencies in the range of $475-495 \mathrm{kHz}$, noting that the transmitted frequency lies between $480 \mathrm{kHz}$ and $490 \mathrm{kHz}$. To determine the parameters of the parallel resonant circuit, the quality factor $[7,11,12,18]$.

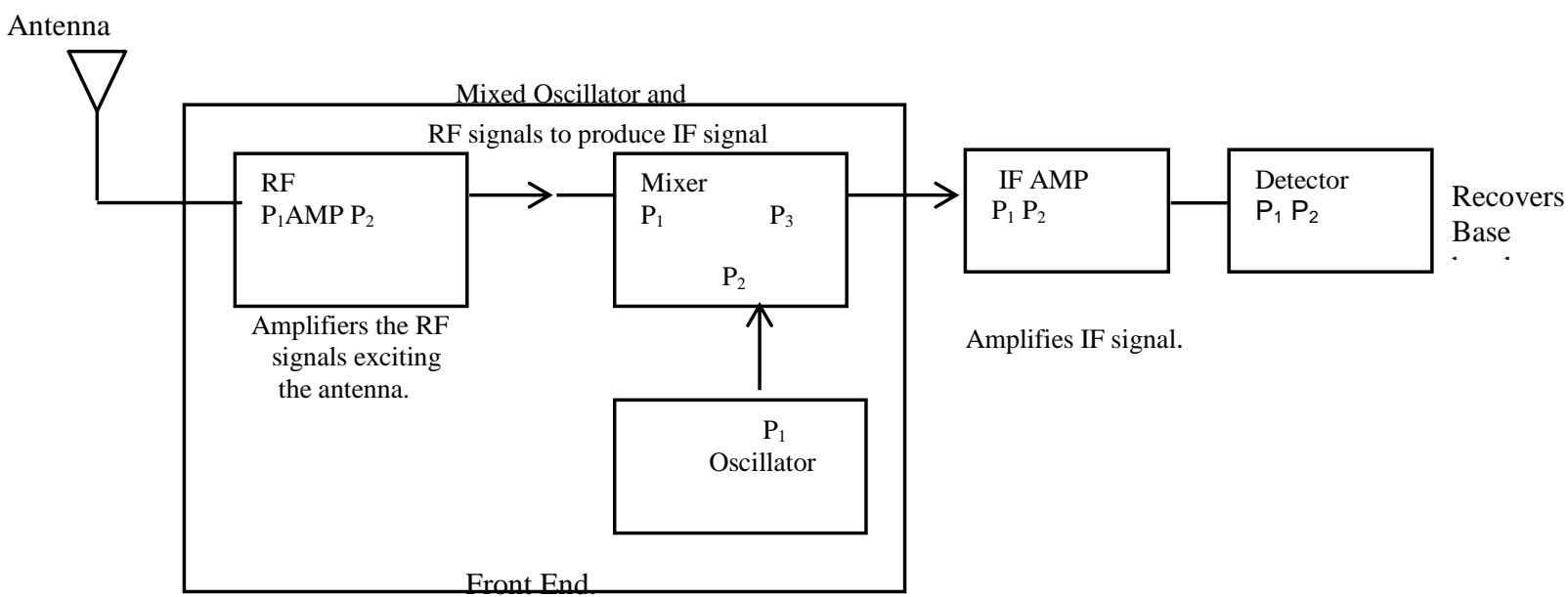

Figure 1: Receiver circuit block diagram.

$\mathrm{Q}$, is $=\mathrm{f}_{\mathrm{R}} / \mathrm{BW}$

where the bandwidth; $B=f_{\mathrm{L} 1}-\mathrm{f}_{\mathrm{L} 2}=495-475 \mathrm{kHz}=20 \mathrm{kHz}$

and, the resonant frequency; $\mathrm{f}_{\mathrm{R}}=\sqrt{ } \mathrm{f}_{\mathrm{L} 1} \mathrm{f}_{\mathrm{L} 2}=485 \mathrm{kHz}$

Thus, $Q$ is $485 / 20=24.3$ which is a narrow band pass filter design specification.

For a parallel resonant circuit,

$$
\mathrm{Q}=\mathrm{Rp} / \mathrm{X}_{\mathrm{L}} \text { or } \mathrm{R}_{\mathrm{P}} / \mathrm{X}_{\mathrm{C}}
$$

where $X_{L}=2 \pi f_{R} L$ and $X_{C}=1 / 2 \pi f_{R} C$, and $R p=Q X_{L}$.

If $\mathrm{L}$ is taken as $10 \mathrm{uH}$, then $\mathrm{X}_{\mathrm{L}}=2 \pi \times 485 \times 10^{3} \times 10 \times 10^{-6}=30.473$. Therefore $\mathrm{Rp}=24.3 \times 30.473=741 \Omega$ or $750 \Omega$.

At resonance, $X_{L}=X_{c}=2 \pi f_{R} L$; thus, $X_{c}=30.473=1 / 2 \pi f_{R} C$ and $C=\left(1 / 2 \pi f_{R}\right) \times 30.473=10.76 n F$

For a tuned amplifier circuit, the gain,

$\mathrm{A}_{\mathrm{v}}=-\mathrm{g}_{\mathrm{m}} \cdot \mathrm{Z}_{\mathrm{L}}$

where $\mathrm{Z}_{\mathrm{L}}$ is the net impedance seen by the drain circuit i.er $/ / \mathrm{QX}_{\mathrm{L}}$, thus,

$\mathrm{Z}_{\mathrm{L}}=1.5 / / 1.5 \mathrm{k} \Omega=0.75 \mathrm{k} \Omega$; and $\mathrm{A}_{\mathrm{v}}=-\mathrm{g}_{\mathrm{m}} . \mathrm{Z}_{\mathrm{L}}=-3.33 \times 10^{-3} \times 0.75 \times 10^{3}=-2.50($ the - ve sign indicates a $180^{\circ}$ phase shift ).

The tuned circuit amplifier calculated values is as shown in Figure 2, with an inductance value of $10 \mu \mathrm{H}$ and a capacitance value of $10 \mathrm{nF}$. This is required to pass a frequency of $480 \mathrm{kHz}$ and $490 \mathrm{kHz}$ freely, and reject any other frequencies surrounding it. A circuit that extracts the original baseband signal from the modulated carrier is called a demodulator or detector. The AM demodulator is the key circuit used for the demodulation of the 
FSK signal used. A simple demodulator circuit for the design is as shown in Figure 3. The diode detector used is basically a half-wave rectifier circuit with a low-pass filter [4,13]. The diode conducts during the positive half cycle of the amplitude-modulated carrier. During the negative half cycle, the diode is reverse biased and no current flows through it or resistor R1. This causes the voltage across resistor R1 to be a series of positive pulses whose amplitude follows the amplitude of the modulated carrier.Capacitor $\mathrm{C} 1$ forms an $\mathrm{R}-\mathrm{C}$ filter with resistor $\mathrm{R} 1$. The operation of the R-C filter can be analyzed in the time domain or the frequency domain $[8,13]$. If we examine the operation of the R-C filter in the frequency domain, it can be considered to be a low-pass filter. This low-pass filter should pass the modulating signal frequencies and reject the carrier frequency and all other harmonic frequencies generated by the nonlinear diode. If the correct value of $\mathrm{C}_{1}$ is selected for a cutoff frequency that is above the modulating frequencies and below the carrier frequency, the waveform across capacitor $\mathrm{C}_{1}$ will be a close approximation to the original modulating signal. If the cutoff frequency is too close to the carrier frequency, the output ripple will be too large. If the cutoff frequency is lower than the modulating frequency, diagonal distortion will result. The purpose of coupling capacitor $\mathrm{C}_{2}$ in the AM diode detector is to remove the dc component from the recovered signal. The coupling capacitor will eliminate this dc component because a capacitor can pass an ac signal, but does not pass a dc voltage. The coupling capacitor $\left(\mathrm{C}_{2}\right)$ and load resistor $\left(\mathrm{R}_{2}\right.$ combination acts like a high-pass filter $[6,8,14]$.

With the use of the tuned circuit design of Figure 2 and the demodulator circuit of Figure 3, the receiver circuit for the medium frequency fsk circuit was developed, which consist of the oscillator circuit, FET amplifier circuit with a tuned circuit, operational amplifiers amplifier circuits, and a comparator as shown in Figure 4. The output from the detector, which is the FSK signal generated at the transmitting end, is fed into two separate band -pass filters; one for mark at $20 \mathrm{kHz}$ and the other for space at $10 \mathrm{kHz}$. To extract the $10 \mathrm{kHz}$ space frequency from the base-band information received after the amplification of the detected signals $[8,12,13,14]$ a band-pass filter network was designed to pass a frequency of $10 \mathrm{kHz}$. To determine the parameters of the parallel resonant band-pass circuit and the quality factor, we use equation 1,2 and 3 to arrive at $Q=f_{R} / B W=$ 100 for a bandwidth of $100 \mathrm{~Hz}$. If $\mathrm{L}$ is taken as $10 \mathrm{uH}$, we get by calculation, $\mathrm{C}=25.36 \mathrm{uF}$ with $\mathrm{Rp}=63 \Omega$.

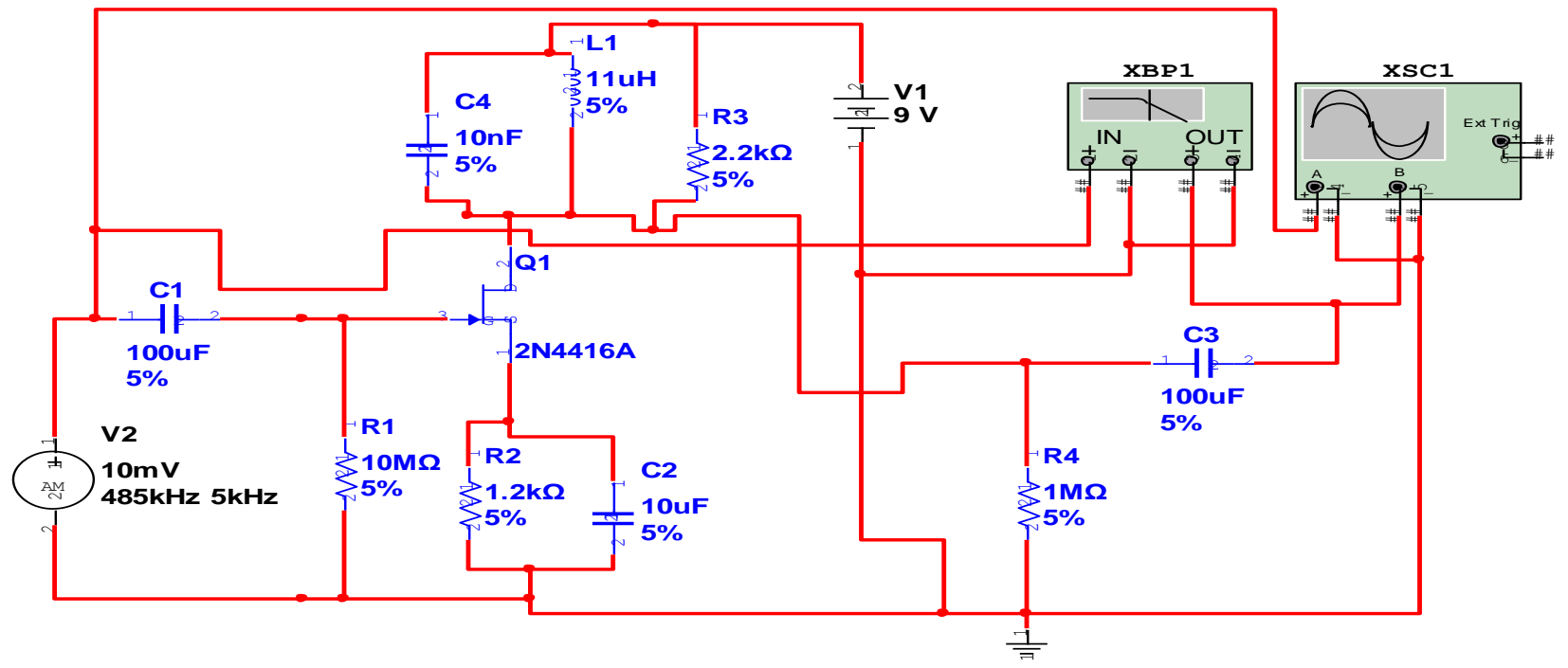

Figure 2: Tuned circuit receiving circuit design.

To extract the $20 \mathrm{kHz}$ mark frequency, a band-pass filter network was designed with the same equations as applied to the extract of $10 \mathrm{kHz}$ information, but here, using a bandwidth of $200 \mathrm{~Hz}$, to get $\mathrm{Q}=100, \mathrm{Rp}=126 \Omega$ and $\mathrm{C}=25.36 \mathrm{uF}$. The output from the parallel filters are fed to a comparator circuit. A comparator is a circuit which compares an input signal $\mathrm{Vi}(\mathrm{t})$ with reference voltage $\mathrm{Vr}$. When the input exceeds $\mathrm{Vr}$, the comparator output voltage ( $\mathrm{Vo}$ ) takes on a value which is very different from the magnitude when Vi is smaller than Vr. If the input to an op-amp comparator is a sine wave, the output is a square wave. If a zero crossing detector is used, (i.e. when $\mathrm{Vr}$ is set to zero), a symmetrical waveform results. The output from the comparators is the digital equivalent of the coded signals sent by the transmitter circuit, and this can be transferred to a microcontroller circuit, to act as a coded signal representing an information from the transmitting end. 


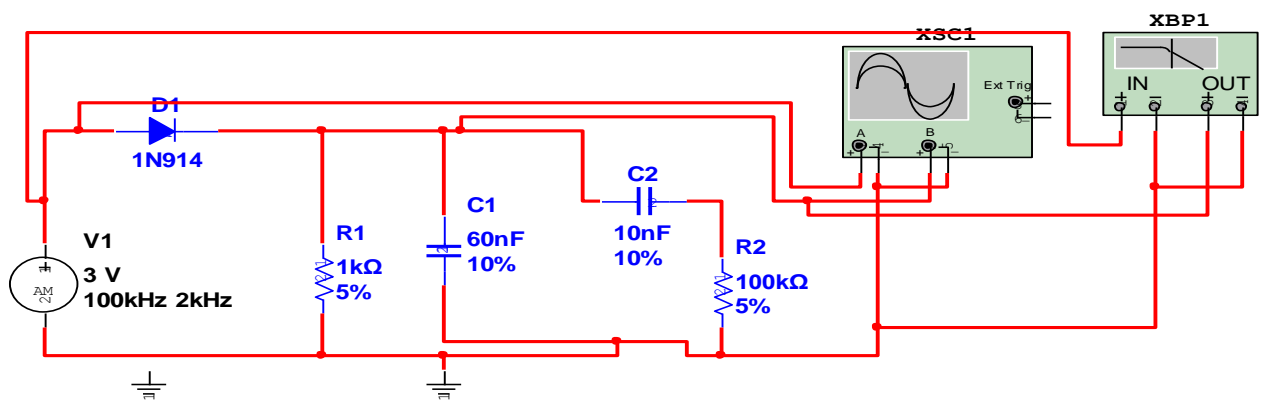

Figure 3: Diode detector circuit diagram

\section{Results of the designed receiver circuit}

The response of the receiving RF amplifier is as shown in Figure 5 giving an output voltage of $660 \mathrm{mV}$, which is further amplified using the common source FET amplifier of Figure 2, to give an output of about 4.5V, as shown in Figure 6. The bode-plot response of the receiver to the incoming signals using a FET tuned circuit is as shown in Figure 7. The peak voltage measured is $4.5 \mathrm{~V}$. This voltage is enough to drive the AM diode detector whose forward voltage response is $0.7 \mathrm{~V}$. The bode-plot response of the receiver to the incoming signals using a FET tuned circuit of in Figure 7 shows that only frequencies above $470 \mathrm{kHz}$ and below $495 \mathrm{kHz}$ are allowed to pass through the network with a resonant frequency of $483.553 \mathrm{kHz}$ and a gain of $27.734 \mathrm{~dB}$, while others are totally attenuated.

The output voltage from the diode detector circuit is $1.32 \mathrm{~V}$, as measured by the oscilloscope, and this voltage is amplified using the FET rf amplifier circuit of Figure 2. The output from the detector is the frequency shift keying signal transmitted from the OTS at a voltage of $8.5 \mathrm{~V}$ and $7.4 \mathrm{~V}$ as shown in Figure 8 . The output from the detector is the frequency shift keying signal transmitted from the OTS at a voltage of $8.5 \mathrm{~V}$ and $7.4 \mathrm{~V}$ as shown in Figure 8. The detector output signal is fed to two separate band - pass filters (one for $10 \mathrm{kHz}$ and the other for $20 \mathrm{kHz}$ ), with their component values calculated to give a response as shown in Figure 6. This shows that the $20 \mathrm{kHz}$ was separated from the $10 \mathrm{kHz}$ signal.

The output of the two separated parallel filters of Figure 9, was fed into a comparator circuit which gives a response shown in Figure 10. When the $20 \mathrm{kHz}$ representing the mark frequency (or the High State of the transmitted information) passes through the circuit, a voltage of $5 \mathrm{~V}$ is achieved and when the frequency is changed to $10 \mathrm{kHz}$, the voltage is in its reference voltage level of OV.

This digital signal forms an input signal to the microcontroller circuit which is in serial form. The serial digital information from the receiver, being fed into the microcontroller is processed and transferred to the on line microcomputer for interpretation. This digital signal can be used for many security information purpose including electronic coding of housing units in a decentralized community for electronic policing of an environment under surveillance, to solve the problems of household breaking -in and stealing, robberies and other crime preventive measures $[15,16,17]$. The reliability of the designed receiver circuit was calculated for a 1 year continuous operating period $[19,20]$, and was found to be $74.7 \%$.

Table 1 : Measured Band pass frequencies and gain for resonant frequency of $20 \mathrm{kHz}$.

\begin{tabular}{|l|l|}
\hline Bandpass frequency in $\mathrm{kHz}$ & Measured gain in $\mathrm{dB}$ \\
\hline 18.29 & -42.037 \\
\hline 19.055 & -37.494 \\
\hline 19.449 & -33.718 \\
\hline 19.851 & -26.789 \\
\hline 20.261 & -23.76 \\
\hline 21.108 & -34.519 \\
\hline 22.445 & -42.401 \\
\hline 23.382 & -45.272 \\
\hline
\end{tabular}




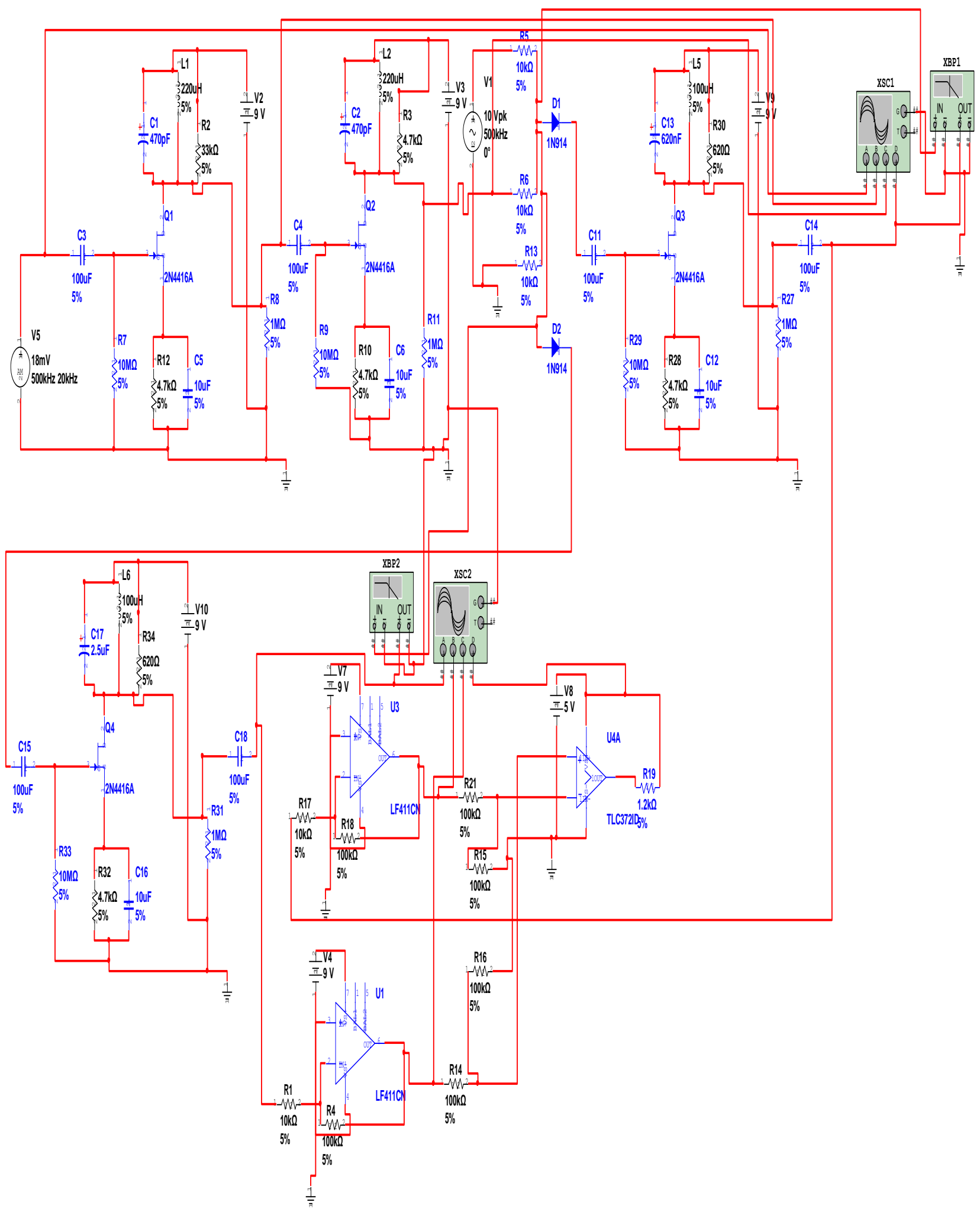

Figure 4:Receiver circuit for the medium frequency fsk circuit. 


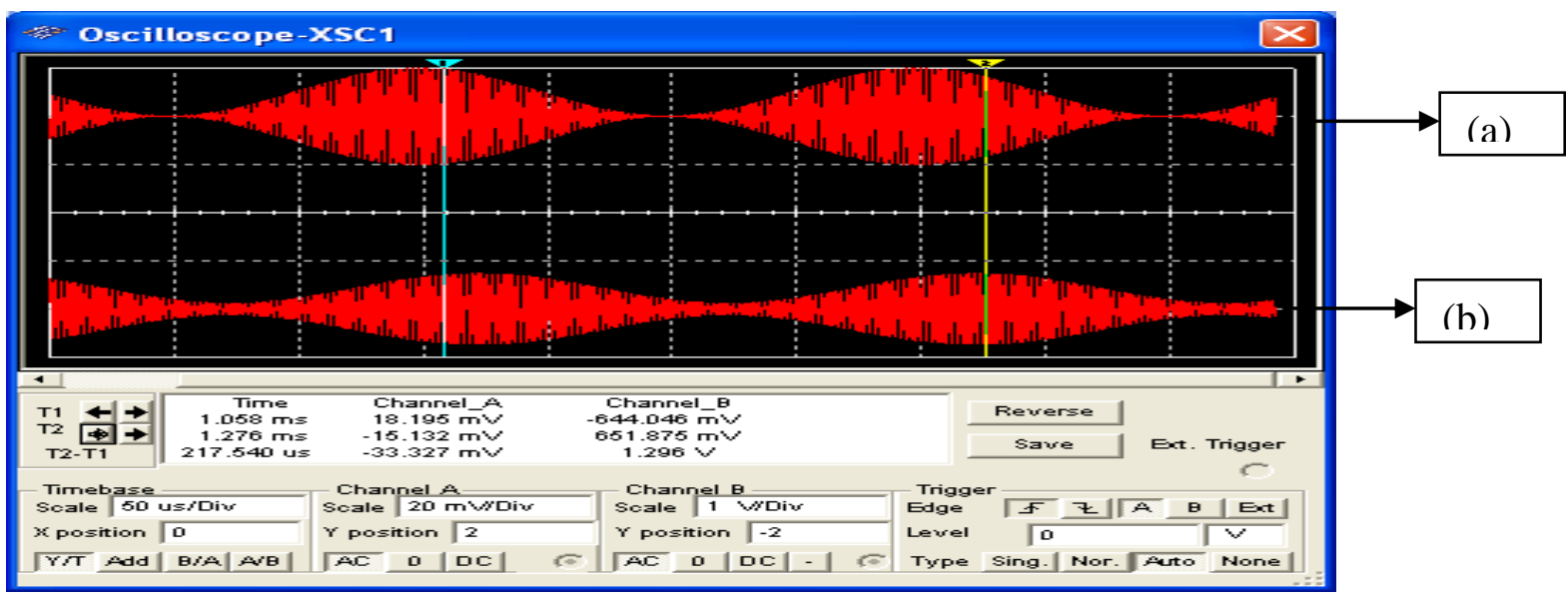

Figure 5: Waveform of the FET receiving amplifier response

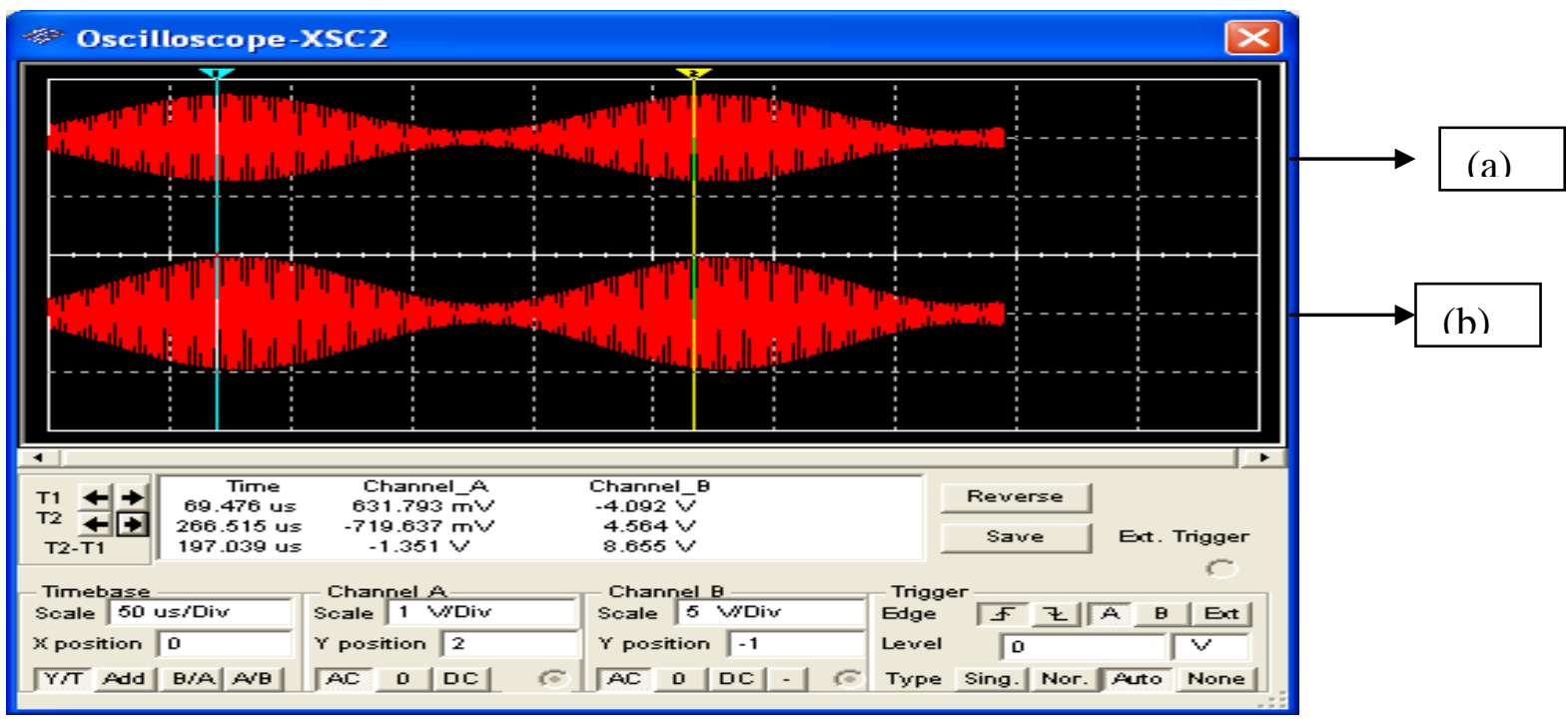

Figure 6: Amplification of RF amplifiers output voltage to 4.5V using FET amplifier.

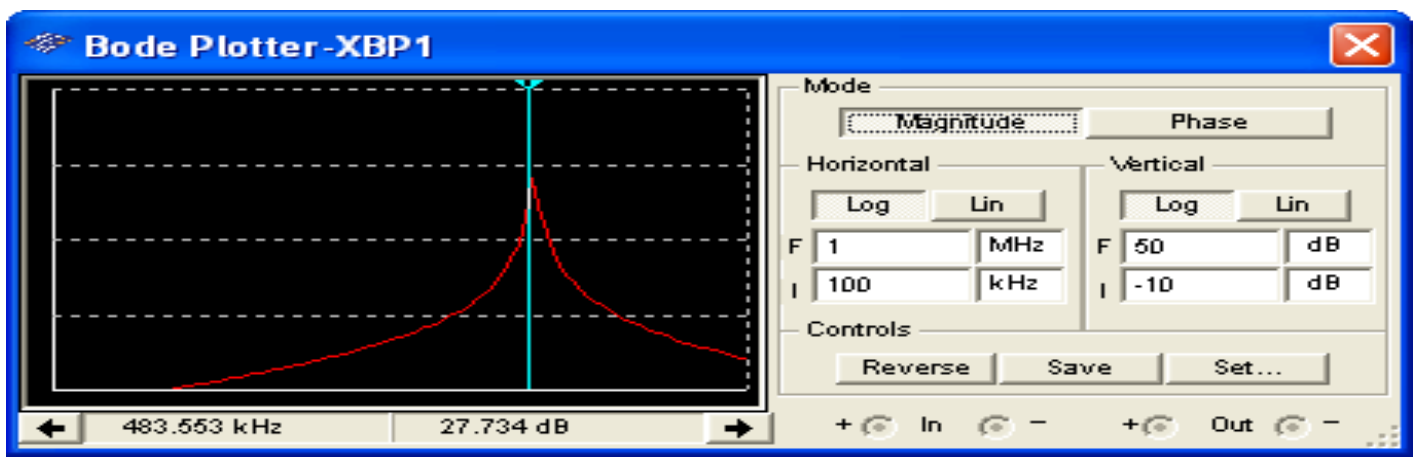

Figure 7: Bode plot of the receiving rf amplifier circuit response 
Development of a receiver circuit for medium frequency shift keying signals.

Table 2: Measured Band pass frequencies and gain for resonant frequency of $10 \mathrm{kHz}$.

\begin{tabular}{|l|l|}
\hline Bandpass frequency in $\mathrm{kHz}$ & Measured gain in $\mathrm{dB}$ \\
\hline 9.12 & -52.385 \\
\hline 9.698 & -48.116 \\
\hline 10.00 & -44.970 \\
\hline 10.312 & -39.583 \\
\hline 10.633 & -19.688 \\
\hline 11.307 & -42.074 \\
\hline 12.023 & -48.954 \\
\hline 14.017 & -56.330 \\
\hline
\end{tabular}

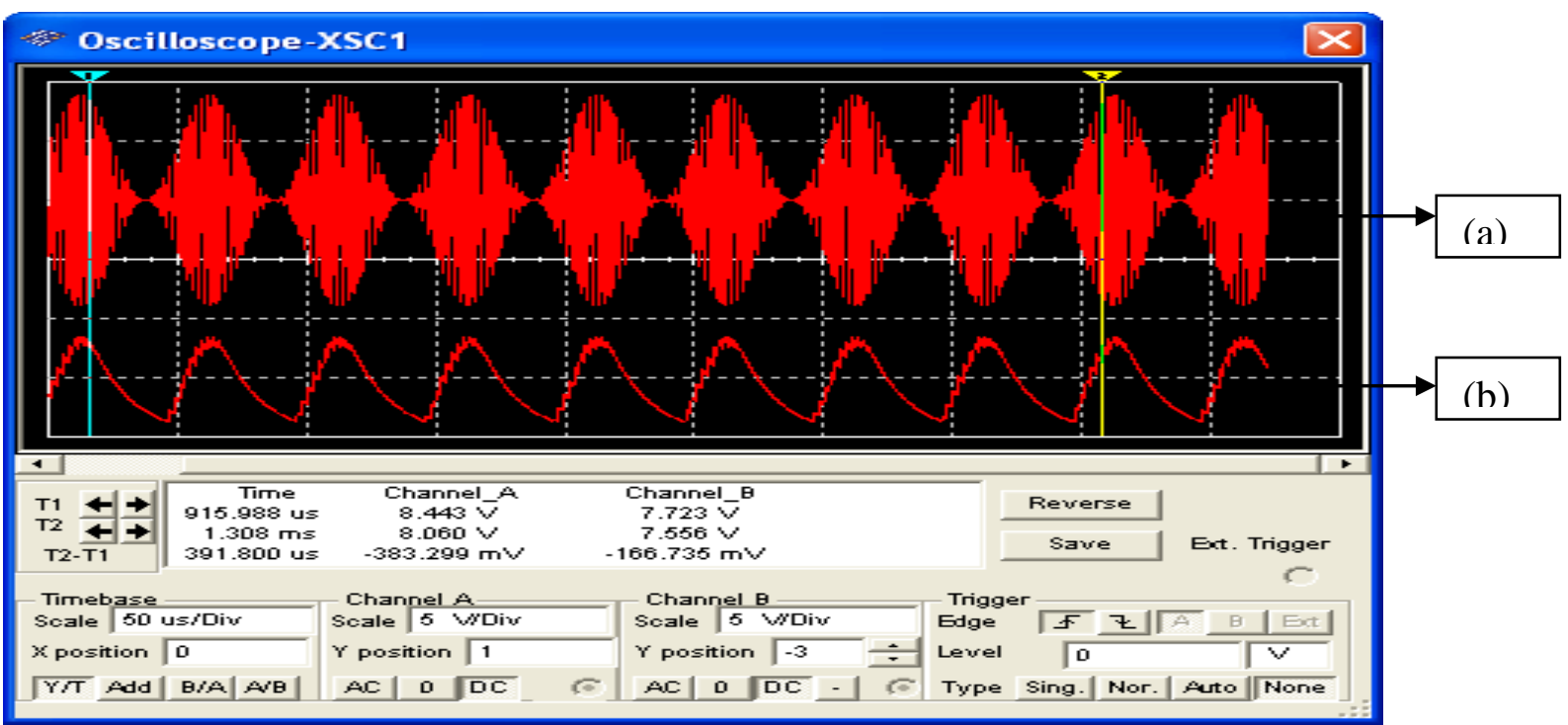

Figure 8: Diode detector output response a). Input b). Output

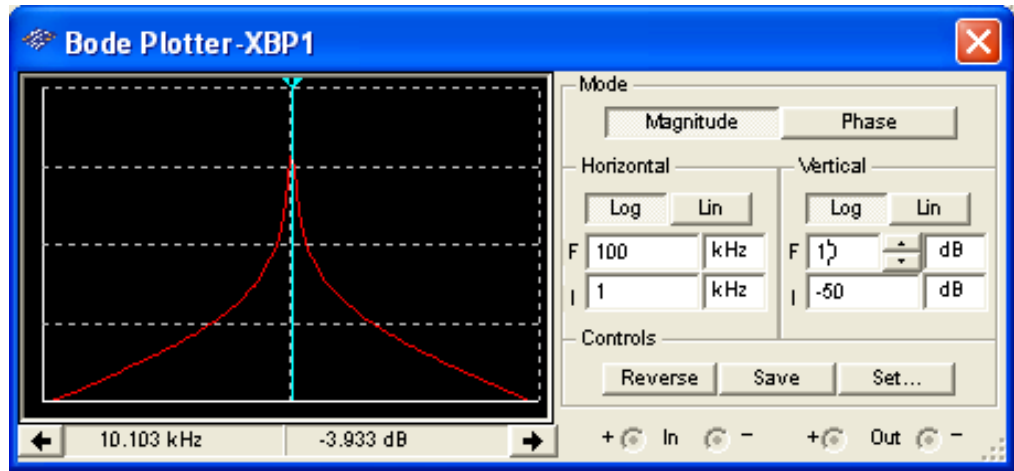

Figure 9: Band -pass network response diagram to extract the $10 \mathrm{kHz}$ signal.

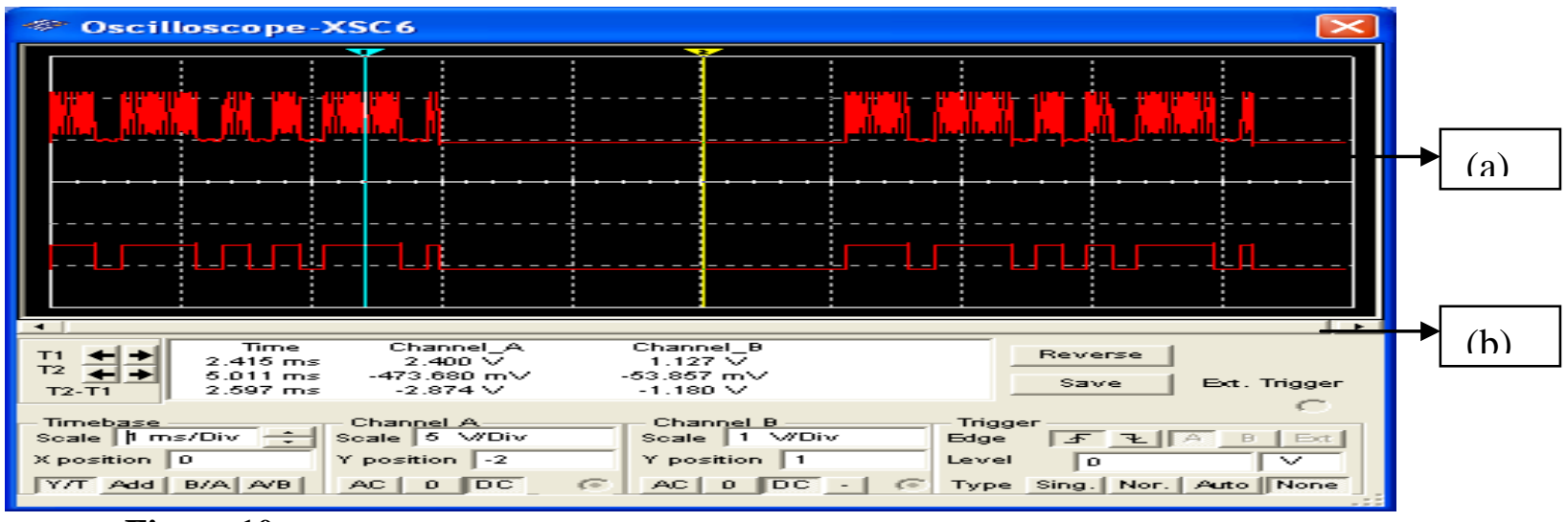

Figure 10: a).Output from the $20 \mathrm{kHz}$ filter, b). The comparators output giving the digital signal 


\section{Conclusion}

The receiver circuit designed receives an fsk signal at $500 \mathrm{kHz}$, the receiving amplifier filters the incoming signal and amplifies the input voltage to a useable level at $4.5 \mathrm{~V}$. The output from the filters through the comparators gives the digital equivalent of the coded signals sent by the transmitter circuit, and this can be transferred to a microcontroller circuit, to act as a coded signal representing information from the transmitting end. The reliability of the designed receiver circuit was calculated for a 1 year continuous operating period, and was found to be $74.7 \%$.This digital signal can be used for many security information purpose including electronic coding of housing units in a decentralized community for electronic policing of an environment under surveillance, to solve the problems of household breaking -in and stealing, robberies and other crime preventive measures.

\section{References}

[1] J.V. Andrew, and K.O. Jim, Principles of digital communication and coding ; Third edition, Prentice Hall international, inc; 2004 .

[2] W.J. Hudson, and J. Luecke, Basic Communications Electronics Radio-Shark R 62-1336, Master Publishing Inc, Lincolnwood, Illinois; 1999

[3] M.L. Anand, Electronic principles - Devices and Circuits, $6^{\text {th }}$ edition, S.Chand and Company, 1999

[4] A.K. Theraja, and B.L. Theraja, A textbook of electrical Technology, $23^{\text {rd }}$ ed., S.Chand and Company, 2003.

[5] J.S. Beasley, and G.M. Miller, Modern Electronic Communication, $8^{\text {th }}$ edition, Pearson Education inc; 2005.

[6] H.R. Berube, Learning Electronics communications through experimentation using electronic workbench multisim; Prentice Hall inc. 2002

[7] R.S. Sheda, A textbook of Digital Electronics, $1^{\text {st }}$ edition, S.Chand and company ltd;.2004

[8] S. Anokh, and A.K. Chabra, Fundamentals of Digital Electronics and Microprocessors; $2^{\text {nd }}$ edition; S. Chand and Company,2003

[9] H. Baggot, Practical filter design, Elektor electronics journal, April, 1989, 22-29

[10] R.F.W. Coates, Modern Communication Systems , $2^{\text {nd }}$ edition, Prentice Hall, New Jersey; 1982

[11] M. Schwartz, Information Transmission, modulation, and Noise. $3^{\text {rd }}$ edition, McGraw-Hill Series in Electrical Engineering, 1980.

[12] J.Milmann, and C.C. Halkias, Integrated Electronics, $30^{\text {th }}$ ed. S.Chand and company ltd;.2004.

[13] R.J. Brad, and E.S. Carlos, Frequency shift keying (FSK) modulator using MEM's switch,2004www.goggle .fsk . Visited on 12 ${ }^{\text {th }}$ February 2006

[14] O.O. Fagbohun, Development of a low cost frequency shift keying (fsk) modulator with transistor switching ; International Journal of Engineering , 4(2), 2010, 249-255

[15] O.O.Fagbohun. Improving the policing system in Nigeria: "Using Electronic Policing", Journal of Engineering and Applied sciences, medwell journals, ISSN 1816-949x, 6(4), 2007, 1223-1228

[16] T. Balogun, Police welfares and state police agitation; $2^{\text {nd }}$ national workshop on security of life and property in Nigeria, Sheraton Hotel Abuja, 2002, 25-26 $6^{\text {th }}$ June

[17] R. Duncan, Revolution in home security; IEE communication Engineering magazine, 2003, 36 -42

[18] A.K. Theraja, and B.L. Theraja, A textbook of electrical Technology, $23^{\text {rd }}$ ed., S. Chand and Company, 2003

[19] C.O. Oroge, Fundamentals of reliability and testing methods. $2^{\text {nd }}$ Edition, Sooji press ltd, 1991

[20] C.O. Oroge, Control System Engineering, 1st edition, University press Plc, 1998 